\title{
A Method for Controlling the Process of Dynamic Settling of Oil Emulsion and $A$ Device for Its Implementation
}

\author{
Rzayev Ab.G. ${ }^{1}$, Asadova R.Sh. ${ }^{1}$, Gurbanov Z.G. ${ }^{2}$, Iskenderova A.T. ${ }^{3}$ \\ 1Institute of Control Systems of ANAS \\ 2SOCAR, Baku Higher Oil School \\ ${ }^{3}$ Baku Secondary School No. 153 \\ E-mail: Abbas_r@mail.ru; Renaasadova2007@rambler.ru; zafer_adna@mail.ru; \\ iskenderova.2705@gmail.com
}

\author{
*Corresponding author details: Asadova R.Sh.; renaasadova2007@rambler.ru
}

\begin{abstract}
The paper describes a method for controlling the process of dynamic settling of oil emulsion (OE). The method involves measuring the flow rate of oil emulsion at the inlet of the settler, the optical density of the fluid layer along the height of the settler, the water level pressure at three points of the height of the settler. The measured values are used to determine the asphaltene content in the fluid layer along the height of the settler and the level of the water cushion (WC) in the settler. The values of these quantities are compared with their nominal ones and when the WC level deviates upwards, the flow rate of drainage water discharged from the settler is increased, and vice versa, and if the asphaltene content deviates upward, the oscillation frequency or redistribution of the initial OE flow among parallel-working settlers is increased. When determining the asphaltene content in the fluid layer, the height of the settler is taken into account. When changing the oscillation frequency is inefficient, if the asphaltene content in the fluid layer increases, the dosage of the demulsifier is increased and vice versa.
\end{abstract}

Keywords: settler; oil emulsion; intermediate emulsion layer; water cushion level; asphaltene-resin components of oil; optical density; hydrostatic pressure

\section{INTRODUCTION}

The paper deals with a problem related to the oil industry, in particular to oil treatment, namely, to the systems of centralized control of the processes of dynamic settling and treatment of oil. The main principles of oil treatment technology were developed on the basis of theoretical and experimental studies formed many years ago [1-9]. The most important components of the oil treatment (OT) process determining its effectiveness, are the removal of reservation sheaths (RS) from emulsified drops of reservoir water and separation of oil and water into phases $[1,2]$. The completeness and speed of these stages are crucial for the required quality of OT process. As we know, the stability of an oil emulsion is determined by sulfonic acids, which are anionic surfactants, mechanical impurities, such as rock components, slightly soluble salts, which, being hydrophobized by asphaltene-resin substances, are concentrated on the RS of water globules, preventing their fusion [3-5].

It is obvious that the necessary and effective impact on RS of emulsified water droplets can be provided by a demulsifier only within the limits of certain indicators of the properties of these RS, which are characteristic of an oil-water emulsion, since the effect of the demulsifier (D) weakens with an increase in the concentration of stabilizing components on the RS surface. These components, as a rule, are substances not related to the components of oil: fine clay, iron sulfide, etc. An increase in their concentration leads to an increase in the mechanical strength of RS.

It is known that the main part of the process of dynamic settling of an oil emulsion is the removal of RS from drops of emulsified reservoir water. Demulsifiers are used to break down RS, but their effective use is limited by subjective reasons. This is especially common for marginal wells with heavily-watered oil. The mechanism of intensification of the process of dynamic settling of oil emulsion is as follows. The redistribution of the flow facilitates cyclic change in the flow rate of oil emulsion in the settlers and the oscillatory motion (compression and expansion) of the IEL, leading to the breakdown of RS, the coalescence of emulsified water droplets and the transfer of mechanical impurities into the water cushion of the settler. As a result, the quality of commercial oil improves (the content of water and mineral salts in the treated oil decreases) and the risk of flooding of the settler decreases. In the settler, where the volume of oil emulsion is greater than the average value (the value for a uniform distribution of the flow of oil emulsion in parallel-working settlers) as a result of cyclic redistribution, the intermediate emulsion layer expands, the kinetic energy increases, and the efficiency of the collision between the drops increases, leading to the disintegration of RS and coalescence of drops. And when the volume of $\mathrm{OE}$ is smaller than the average value, the intermediate emulsion 
layer is compressed, the distance between the drops decreases, leading to the coalescence of the drops, increasing the efficiency of oil treatment. It is also known that the efficiency of dynamic settling is affected by the WC level and the change in the asphaltene concentration. The higher the asphaltene concentration and the WC level, the worse the process of dynamic settling of oil emulsion. This is when the dosage of the demulsifier and the frequency of flow redistribution should be increased, and vice versa.

From literature, we know of various methods and systems that implement the processes of oil treatment to commercial standards. There is a method to control the process of thermochemical dehydration of oil [10], which consists in automatic adjustment of the dosage of surfaceactive substances (surfactants) based on the water content in oil emulsion for its breakdown and allows controlling the process of breakdown of oil emulsion during the treatment of commercial oil to the standard. However, this method does not take into account the formation of an intermediate emulsion layer (IEL) in the settler. IEL consists of emulsified water droplets with reservation sheaths formed by mechanical impurities and natural emulsifiers (surfactants) adsorbed on water and oil droplets and preventing their coalescence. This is especially typical for a settler operating in a dynamic mode, which affects the quality of the treated commercial oil. There is a known [11] method for settling an oil emulsion and a system for controlling the process of thermochemical treatment of oil. The method consists in measuring the differential pressure along the height of the settler, measuring the flow rate of oil emulsion, automatic dosing of the demulsifier based on the flow rate of oil emulsion, and creating an artificial oscillatory mode between the settlers by redistributing the flow of oil emulsion among the parallel-working settlers. The control of the uneven distribution of the total volume of the incoming oil emulsion among the parallel-working settlers is carried out through the actuators installed on the oil emulsion inlet line of each settler. The signal to start the control is the value of the control action calculated on the basis of the measured data by the algorithm presented in the paper. The method makes it possible to improve the process of breakdown of oil emulsion and adjust the dosage of the demulsifier. But this method does not allow estimating the volume of the intermediate layer of oil emulsion and the level of the water cushion in the settler, an excessive increase in which can lead to the flooding of the settler. The latter worsens the breakdown process, rendering the system incapable to control the process efficiently.

This paper discusses and proposes, on the basis of the known work [11], a method and system that allow improving the quality of the process of dynamic settling of oil emulsion. To control the water cushion level and manage the process, the WC level is measured by the hydrostatic method, and the differential pressure along the height of the settler is measured at three points. On the basis of the measurements obtained, the authors developed an algorithm and determined the control indicator for the process of dynamic settling of oil emulsion.

Depending on the deviation of the value of the controlled indicator (the factor of quality of the dynamic settling of $\mathrm{OE}$ ) from the nominal value for the i-th settler, the WC level and the $\mathrm{OE}$ flow rate in that settler are changed. If the calculated value of the control indicator from the nominal one increases, the $\mathrm{WC}$ level and the $\mathrm{OE}$ flow rate are decreased and the specific flow rate of the demulsifier is increased.
The essence of the method consists in controlling the process of dynamic settling of oil emulsion. It involves measuring the differential pressure at three points along the height of the settler, the flow rate of oil emulsion, the concentration of asphaltene-resin components of oil in settlers, the content of water in oil emulsion at the inlet of the settler, the optic density of the oil layer, the level of the water cushion in the settler, adjusting the dosage of demulsifier and redistributing the flow of oil emulsion among parallel-working settlers. The value of the control action is calculated from the measured data. The system for controlling the process of dynamic settling of oil emulsion contains pressure transducers along the height of the settler, oil emulsion and demulsifier flow gauge, actuators on the flow line at the inlets of settlers and the drainage water discharge line, infrared radiation and reception sensors and a control and indication unit. The control unit implements control action on the actuators based on the value of control action, which is calculated by the following algorithm:

$$
\begin{gathered}
W_{11-7}=\frac{\rho_{o e}-\rho_{o}}{\rho_{w}-\rho_{o}}=\frac{\frac{\Delta P_{1-7}}{g h}-\rho_{o}}{\rho_{w}-\rho_{o}} \\
W_{7-13}=\frac{\frac{\Delta P_{2-3}}{g h}-\rho_{o}}{\rho_{w}-\rho_{o}} \\
\left.h_{w}{ }^{m}=W_{11-7}+W_{7-13}\right) \frac{1}{2} h \\
\Delta h=h_{w}{ }^{m}-h_{w}^{p} \\
D=\lg \left(\frac{J_{0}}{J_{1}}\right) \\
Z_{A}=a D / l=15,813 D \\
Z_{A}=Z_{A}^{c}-Z_{A}^{p}
\end{gathered}
$$

where $W_{-}(11-7), W_{-}(7-13)$ are the content of water in the fluid layer along the height of the settler between the tapping points, respectively, fractional;

P_1, P_2, P_3 are the pressure at three measurement points, respectively, kgf/m2; $\rho_{-} w, \rho_{-} \mathrm{o}, \rho_{-} \mathrm{oe}$ are the density of water, oil and $\mathrm{OE}$, respectively, $\mathrm{kg} / \mathrm{m} 3$; $\mathrm{D}$ is the optical density of the fluid between the sensor and receiver at the length of the infrared radiation (IRR) wave, fractional; Z_A is the concentration of asphaltenes in the oil layer, fractional; $Z_{-} A^{\wedge} c, Z_{-} A^{\wedge} p$ are the calculated and the prescribed values, respectively, of the asphaltene concentration in the oil layer; J_0 and J_1 are the intensity of incident infrared radiation and radiation passing through the fluid layer along the height of the settler; $l$ is the height of the settler, m; h_w is the level of the water cushion (of the oil-water phase divide); a is the empirical coefficient, determined experimentally; 『 h_w 『 ${ }^{\wedge} \mathrm{m}, \mathrm{h} \_\mathrm{w}^{\wedge} \mathrm{p}$ are the measured and the prescribed (nominal) values, respectively, of the level of WC in the settler, $\mathrm{m}$.

When $\Delta Z_{-} A>0$, the oscillation frequencies of IEL are increased, and vice versa, when $\Delta \mathrm{Z}_{-} \mathrm{A}<0$, the oscillation frequencies of IEL are decreased. When $W_{-}$(11-7)/W_ (713) $=0$ and $\Delta Z_{-} A=0$, the oscillation goes into the mode of uniform distribution of the IEL flow between the settlers, and when $\Delta \mathrm{h}>0$ it increases the drainage water flow rate and vice versa. 
The proposed method and control system make it possible to quickly and reliably control and efficiently manage the process of dynamic settling of oil emulsion in settlers to obtain the required quality of commercial oil.

The system implementing the method is illustrated in Fig. 1 , showing a schematic diagram of the system for controlling the dynamic settling of oil emulsion (OE), which contains: 1 - OE flow rate gauge; 2 - converter of the flow rate gauge signal; $31-3 \mathrm{l}$ - sensors and 4 and $4 \mathrm{n}-$ IRR receivers; 51 and $5 n, 61$ and $6 n$ - converter of the IRR sensor signal; 9 - demulsifier level sensor; 10 - converter of the demulsifier level sensor signal $71,111,121 \ldots, 7 \mathrm{n}$, $11 n, 12 n$ - pressure gauges; $81, \ldots, 8 n, 131, \ldots, 13 n-$ converters of the pressure gauge signal; 22 - centrifugal OE feed pump; 14 - demulsifier distribution plunger pump; 15 - control and indication unit; $161 \ldots 16 \mathrm{n}$ - settlers; $171, \ldots, 17 \mathrm{n}-$ OE flow rate at inlets $161, \ldots, 16 \mathrm{n} ; 181, \ldots, 18 \mathrm{n}$, 19 - actuators; 20 - demulsifier tank. Process lines: I - OE line at the inlet to the settler; II - drainage water line; III $\mathrm{OE}$ line at the centrifugal pump inlet; IV - demulsifier line; $\mathrm{V}$ - dehydrated oil line.

The system works as follows.

All gauges are connected to the control and indication unit (CIU) 15 with a given frequency via corresponding converters and scanned, and based on the values of the received signals, the control action is calculated by the proposed algorithm. If the value of the controlled indicator exceeds the nominal value, through the action of the signal from the CIU on the corresponding actuators, the specific flow rate (dosage) of the demulsifier, the frequency of redistribution of the flow of oil emulsion among the parallel-working settlers and the level of the water cushion are decreased. If the value of this indicator is less than the nominal one, the dosage of the demulsifier and the frequency of redistribution of the oil emulsion flow are decreased and the water cushion level are increased. The nominal value of the control action is determined as the weighted average value from the previously accumulated indicators.

The technical effect of the proposed method is that it allows quickly and reliably controlling and efficiently managing the process of dynamic settling of oil emulsion in parallel-working settlers to obtain the required quality of commercial oil.

\section{CONCLUSION}

A method has been developed to control the processes of thermochemical treatment of oil, which consists in breaking down the reservation sheaths of emulsified water drops in OE and dynamic settling (DS) of emulsified water droplets, affecting the dosage of a demulsifier (chemical) and actuators installed on the drainage water lines and at the inlets of settlers in the OE supply line, in order to create an oscillatory regime in settlers (uneven distribution of the flow of OE among settlers). New formulas (algorithms) for calculating the main indicators of the DS process and a device for implementing the method have been developed.

\section{REFERENCES}

[1] Pozdnyshev G.A. Stabilizing and breaking oil emulsions, Moscow, Nedra, 1982 (in Russian)

[2] Methods for stabilizing the operation of oil treatment units F.R., Gubaydullin, T.F. Kosmacheva, V.P. Tronov (et al.) // Moscow, Neftyanoye Khozyaystvo, 2003, No 2, pp. 66-68 (in Russian)

[3] Reasons for increasing the stability of oil-water emulsion/ R.Z. Sakhabutdinov, T.F. Kosmacheva, F.R. Gubaydullin, O.S. Tatianina // Moscow, Neftyanoye Khozyaystvo, 2007, No 1, pp. 74-77 (in Russian)

[4] Rzayev Ab.G. The scientific foundations for calculating the design and management of processes of separation of oil emulsion in oil treatment and oil refining: dissertation for the degree of $\mathrm{PhD}$ in Engineering. - Baku: ITPHT, 1994 (in Russian)

[5] Aliev T.A., Guluyev G.A., Rzayev Ab.G. Mathematical models of the intermediate emulsion layer in the settlers of the thermochemical oil treatment plant // Moscow, Neftepererabotka i Neftekhimiya, 2011, No 8, pp. 50-53 (in Russian)

[6] Baykov N.M., Pozdnyshev G.N., Mansurov R.I. Collection and field treatment of oil, gas and water. Moscow, Nedra, 1981, 261 p. (in Russian)

[7] A.C. 1430593. Method of automatic control of the demulsifier dosing process at the oil treatment plant / M.A. Mekhtiev, Ab.G. Rzayev, V.I. Loshenov, G.A. Kasper, O. M. Pak // BI., 1984, No 47

[8] Rzayev Ab.G., Rasulov S.R., Abasova I.A. Automatic regulation of the desmulsifier dosing process at a unified oil treatment unit // Baku, Vestnik Azerbaydzhanskoy Inzhenernoy Akademii, 2013, No 3, p. 75-79 (in Russian)

[9] A.C. USSR 18055130. Method of controlling oil dehydration processes / Ab.G. Rzayev // Moscow, BI, 1993, No 12 (in Russian)

[10] System for automatic treatment and dosage of surfactants at oil treatment units, Avtomatizatsiya i Telemekhanizatsiya Neftyanoy Promyshlennosti, 1975, No 4, pp. 8-10 (in Russian)

[11] Development of a system for control and management of thermochemical oil treatment processes, Avtomatizatsiya i Telemekhanizatsiya Neftyanoy Promyshlennosti, Moscow, 2013, No 12, pp. 38-41 (in Russian) 